\title{
Cosmological models with Gurzadyan-Xue dark energy
}

\author{
G. V. Vereshchagin $\ddagger$ and G. Yegorian $\S$ \\ ICRANet, P.le della Repubblica 10, I65100 Pescara, Italy and ICRA, Dip. Fisica, \\ Univ. "La Sapienza", P.le A. Moro 5, I00185 Rome, Italy
}

\begin{abstract}
The formula for dark energy density derived by Gurzadyan and Xue is the only formula which provides (without a free parameter) a value for dark energy density in remarkable agreement with current cosmological datasets, unlike numerous phenomenological scenarios where the corresponding value is postulated. This formula suggests the possibility of variation of physical constants such as the speed of light and the gravitational constant. Considering several cosmological models based on that formula and deriving the cosmological equations for each case, we show that, in all models source terms appear in the continuity equation. So, on the one hand, GX models make up a rich set covering a lot of currently proposed models of dark energy, on the other hand, they reveal hidden symmetries, with a particular role of the separatrix $\Omega_{m}=2 / 3$, and link with the issue of the content of physical constants.
\end{abstract}

PACS numbers: $98.80 \mathrm{Cq}, 06.20 \mathrm{fn}$

$\ddagger$ e-mail address: veresh@icra.it

$\S$ e-mail address: gegham@icra.it 


\section{Introduction}

With the establishment of observational evidence favoring dark energy domination in the recent history of the cosmological expansion, numerous different models were suggested to account for it. For most of them the value of the corresponding density parameter is chosen to fit the observational one. In contrast, Gurzadyan and Xue formula [1] for dark energy predicts the observed value for the density parameter of the dark energy. The formula reads [1, 2

$$
\rho_{G X}=\frac{\pi}{8} \frac{\hbar c}{L_{p}^{2}} \frac{1}{a^{2}}=\frac{\pi}{8} \frac{c^{4}}{G} \frac{1}{a^{2}},
$$

where $\hbar$ is the Planck constant, the Planck length is $L_{p}=\left(\frac{\hbar G}{c^{3}}\right)^{\frac{1}{2}}, c$ is the speed of light, $G$ is the gravitational constant, $a$ is the scale factor of the Universe. Following the original idea by Zeldovich [3], it corresponds to the cosmological term

$$
\Lambda_{G X}=\frac{8 \pi G \rho_{G X}}{c^{2}}=\frac{8 \pi G}{c^{2}} \frac{\pi}{8} \frac{c^{4}}{G} \frac{1}{a^{2}}=\pi^{2}\left(\frac{c}{a}\right)^{2} .
$$

Therefore, to keep it constant, the speed of light should vary with cosmological expansion as $c \propto a$. One can consider other possibilities as well, assuming the presence of some fundamental physical quantity and admitting variation of basic physical constants [4] in the spirit of Dirac approach. Since the Planck constant does not appear in (11), these can be the gravitational constant and the speed of light.

In the literature there is a long-standing discussion how to understand possible variability of physical constants, linking this point to the meaning of world constants and units in physics [5. Clearly experimentally only dimensionless constants variation can be detected, but the meaning and the content of the underlying physical theory changes depending on the issue which dimensionful quantity varies.

In fact, models with varying physical constants are among the currently discussed ones (e.g. [6]). However, models based on the formula (11) are very different from various phenomenological models of dark energy, phantoms etc., because of the underlying empirical basis, namely agreement between predictions for dark energy density parameter and the value, following from the array of recent observations.

The classification of various cosmological models following from GX-formula is given in [4. Furthermore, it is shown [7] that GX models provide indeed good fit to supernovae and radio galaxies data. These models contain hidden invariance, the separatrix dividing cosmological solutions into two classes: singular Friedmannian-type and non-singular with vanishing initial density [7, 8]. In this paper we provide cosmological equations for the simplest cases discussed in [4] and generalize them, including radiation field, and compare them to the standard cosmological model.

In the section 2 cosmological framework with varying physical constants is formulated. In the section 3 we derive cosmological equations for particular cases, discussed in [4. We provide analytical solutions for energy density in all models, and also in some models for the scale factor. Conclusions follow in the last section. 


\section{Cosmological equations with varying constants}

In this section we are going to derive cosmological equations, assuming varying constants, such as the speed of light $c(t)$, the gravitational constant $G(t)$ and the cosmological term $\Lambda(t)$ depending on the cosmic time $t$. Einstein equations are postulated to be valid in such a case.

We start from the FRW interval

$$
d s^{2}=-c(t)^{2} d t^{2}+a^{2}(t)\left[\frac{d r^{2}}{1-k r^{2}}+r^{2}\left(d \theta^{2}+\sin ^{2} \theta d \varphi^{2}\right)\right],
$$

where $r, \theta, \varphi$ are spatial coordinates, $a$ is the scale factor. Using Einstein equations

$$
R_{\mu \nu}-\frac{1}{2} R g_{\mu \nu}-\frac{\Lambda}{c^{2}} g_{\mu \nu}=\frac{8 \pi G}{c^{4}} T_{\mu \nu},
$$

and the energy-momentum tensor

$$
T_{\nu}^{\mu}=\operatorname{diag}(-\epsilon, p, p, p),
$$

with $\rho$ being the energy density, $p$ being the pressure, we arrive at the cosmological equations

$$
\begin{aligned}
& H^{2}+\frac{k c^{2}}{a^{2}}-\frac{\Lambda}{3}=\frac{8 \pi G}{3 c^{2}} \rho, \\
& \dot{H}+H^{2}-\frac{\Lambda}{3}=-\frac{4 \pi G}{3 c^{2}}(\rho+3 p)+H \frac{\dot{c}}{c},
\end{aligned}
$$

where $k$ is the sign of spatial curvature, $H \equiv d \ln a / d t$ is the Hubble parameter, a dot denotes time derivative. From (4) and (5) (or from $\frac{8 \pi G}{c^{4}} T_{; \nu}^{\mu \nu}=0$ ) continuity equation can be written

$$
\dot{\rho}+3 H(\rho+p)=-\dot{\rho}_{\Lambda}+\left(\rho+\rho_{\Lambda}\right)\left(4 \frac{\dot{c}}{c}-\frac{\dot{G}}{G}\right),
$$

where have we introduced $\rho_{\Lambda}=\Lambda c^{2} /(8 \pi G)$. The same equations were derived e.g. in [9]. The difference with [9] is that we consider the scaling (II) not as a phenomenological, but as an empirical relation.

It is easy to check that (41) is the first integral of (5I) as it is within standard Friedmann cosmology. Cosmological equations for the case when $c$ and $G$ both depend on time can be found in [10]. For the case when instead $\Lambda$ and $G$ depend on time these equations are given in [1]. Our equations reduce to equations of [10] and 11] with the corresponding assumptions. There is a little difference with [10], because we postulated only invariance of the Einstein equations, while authors of [10] assumed that both Einstein and Friedmann equations are still valid. With these assumptions the last term in (5]) disappears and our equations looks identical to those of [10].

Note, that the former energy conservation does not hold any more: there are sources and drains of energy (e.g. from dark energy to the usual matter, from the variation of the speed of light and the gravitational field strength) in (6). As we will see in the next

section, (6) can be integrated to obtain energy density as explicit function of the scale 
factor. Further for some models Friedmann equation (4) can be explicitly integrated to get the scale factor as a function of time.

In 4] five different cases have been discussed, namely:

(i) $\mathrm{c}=$ const, $\mathrm{G}=$ const, $\Lambda \neq$ const;

(ii) $c \propto a, \mathrm{G}=\mathrm{const}, \Lambda=\mathrm{const}$;

(iii) c=const, $G \propto a^{-2}, \rho_{\Lambda} / c^{2}=$ const, $\Lambda \neq$ const (case 3.1);

(iv) $c \propto a^{1 / 2}, \mathrm{G}=$ const, $\rho_{\Lambda}=$ const, $\Lambda \neq$ const (case 4.2 );

(v) $c \neq$ const, $G \neq$ const, $\Lambda \neq$ const.

In what follows we analyze each of the first four cases, provide cosmological equations and corresponding solutions for them.

\section{Cosmological models based on Gurzadyan-Xue formula}

In this section we present cosmological equations for each of the cases (i-iv) and discuss the corresponding cosmological scenarios, providing analytical solutions, where possible.

\subsection{Models with dark energy and pressureless matter}

In this subsection we put the pressure in (51) and (6) to zero and introduce mass density $\mu=\rho / c^{2}$. Each solution can be parametrized by the usual matter density

$$
\Omega_{m} \equiv \frac{\mu_{0}}{\mu_{c}} \equiv \frac{8 \pi G_{0}}{3 H_{0}^{2}} \mu_{0}
$$

where $\mu_{c}$ is the critical density in usual Friedmannian models, subscript 0 denotes the value of each quantity today. In what follows, to define density parameters we use normalization condition

$$
\sum_{i} \Omega_{i}=1
$$

where $i$ numbers components such as dark energy, radiation, usual matter.

In order to get density as a function of the scale factor we rewrite (6) as

$$
\frac{d \mu}{d a}+3 \frac{\mu}{a}=\mathrm{R} \equiv \frac{1}{4 \pi G} \frac{d c}{c d a}(8 \pi G \mu+\Lambda)-\mu \frac{d G}{G d a}-\frac{1}{8 \pi G} \frac{d \Lambda}{d a} .
$$

The crucial feature of these models is the presence of a separatrix in solutions for the density as a function of the scale factor (see [7] and [8]). This separatrix divides solutions in two classes: the one with Friedmannian singularity, and the one with vanishing density and non-zero scale factor in the beginning. 
To see the difference from our models and the corresponding Friedmannian ones we compute for each case mass transfer rate, namely the ratio between $\mathrm{R}$ of (9) and expansion term $3 \mu / a$, i.e.

$$
\mathcal{M} \equiv \frac{a R}{3 \mu} .
$$

If dimensionless $\mathcal{M}$ vanishes, it means the usual Friedmannian solution $\mu \propto a^{-3}$ holds. When $\mathcal{M}=1$ mass transfer cancels the effect of expansion and the mass density does not change in course of expansion. If it is large, effect of expansion can be neglected.

Model 1 (varying cosmological constant). Neither the speed of light nor the gravitational constant vary with time, but in this case from (2) we find $\Lambda \propto a^{-2}$. Cosmological equations are

$$
\begin{aligned}
& H^{2}+\frac{k^{\prime} c^{2}}{a^{2}}=\frac{8 \pi G}{3} \mu, \\
& \dot{\mu}+3 H \mu=\frac{\pi}{4 G}\left(\frac{c}{a}\right)^{2} H,
\end{aligned}
$$

where $k^{\prime}=k-\frac{\pi^{2}}{3}$. It is clear that $k^{\prime}<0$, i.e. the effect of $\Lambda$ variation is to make spatial geometry negatively curved, even if the original sign of $k$ is " + ". The source term in the continuity equation can be interpreted as the energy transfer from the dark energy component into the matter component (see [11] and references therein).

Solution for the density is given by

$$
\mu(a)=\mu_{0}\left(\frac{a_{0}}{a}\right)^{3}+\frac{\pi}{4 G} \frac{c^{2}}{a^{2}}\left(1-\frac{a_{0}}{a}\right),
$$

and after substitution of this solution to (4) we obtain

$$
\begin{aligned}
\pm A_{1}^{\frac{3}{2}} t=\sqrt{a A_{1}\left(a A_{1}+B_{1}\right)}- & \sqrt{a_{0} A_{1}\left(a_{0} A_{1}+B_{1}\right)}+ \\
& +B_{1} \log \left(\frac{\sqrt{a_{0} A_{1}}+\sqrt{a_{0} A_{1}+B_{1}}}{\sqrt{a A_{1}}+\sqrt{a A_{1}+B_{1}}}\right)
\end{aligned}
$$

where

$$
A_{1}=c^{2}\left(\pi^{2}-k\right), \quad B_{1}=\frac{2 \pi}{3} a_{0}\left(4 G \mu_{0} a_{0}^{2}-\pi c^{2}\right) .
$$

This solution reduces to a simple form for separatrix value with the condition $B_{1}=0$, namely

$$
a=a_{0}+\sqrt{A_{1}} t
$$

The separatrix solution is given by the density parameter $\Omega_{m} \approx 2 / 3$ and depends slightly on the spatial curvature. For high values of the density parameter solutions practically coincide with the corresponding Friedmannian ones. In such a case one can neglect the source term in the continuity equation. This also can be done for large scale factors since solutions approach the separatrix where they describe Friedmann Universe with negative curvature. 
The present scale factor is well defined quantity even for $k=0$ models,

$$
a_{0}=\left(-k^{\prime}\right)^{\frac{1}{2}} \frac{c}{H_{0}} \frac{1}{\sqrt{1-\Omega_{m}}},
$$

since this is the model with effective negative curvature with $\Omega_{k}=-\frac{k^{\prime} c^{2}}{a_{0}^{2} H_{0}^{2}}$. With this definition (8) is satisfied along with with (77).

Mass transfer rate (10) for this model in terms of density parameter is given by

$$
\mathcal{M}=\frac{2 \pi^{2}}{9 k^{\prime}}\left(\frac{1}{\Omega_{m}}-1\right),
$$

and it is clear that $\mathcal{M}$ gets infinite with $\Omega_{m} \rightarrow 0$, i.e. vacuum mass transfer dominates expansion, and in the opposite case the model approaches Friedmannian matter dominated one with negative curvature.

Model 2 (varying speed of light). To keep interpretation of $\Lambda$ as a cosmological constant, we have to require the varying speed of light, $c=\left(\frac{\Lambda}{\pi^{2}}\right)^{1 / 2} a$. At the same time the gravitational constant does not change, $G=$ const. Cosmological equations reduce to the following system

$$
\begin{aligned}
& H^{2}-\frac{\Lambda^{\prime}}{3}=\frac{8 \pi G}{3} \mu, \\
& \dot{\mu}+3 H \mu=\frac{3 H}{4 \pi G}\left(H^{2}+\frac{k \Lambda}{\pi^{2}}\right)=3 H \mu\left(1+\frac{\Lambda}{8 \pi G \mu}\right),
\end{aligned}
$$

where $\Lambda^{\prime}=\Lambda\left(1-\frac{3 k}{\pi^{2}}\right)$. The source in the continuity equation now comes from the speed of light variability.

There is complete analytical solution for this model

$$
\begin{aligned}
& \mu(a)=\mu_{0} \frac{a_{0}}{a}+\frac{\Lambda}{4 \pi G}\left(1-\frac{a_{0}}{a}\right) \\
& a(t)=-\frac{A_{2}}{2 B_{2}}+\frac{A_{2}+2 a_{0} B_{2}}{2 B_{2}} \cosh \left(t \sqrt{B_{2}}\right) \pm \sqrt{\frac{a_{0}}{B_{2}}\left(A_{2}+a_{0} B_{2}\right)} \sinh \left(t \sqrt{B_{2}}\right),
\end{aligned}
$$

where

$$
A_{2}=\frac{2}{3} a_{0}\left(4 \pi G \mu_{0}-\Lambda\right), \quad B_{2}=\Lambda\left(1-\frac{k}{\pi^{2}}\right) .
$$

In this model the separatrix is given by $\Omega_{m}=2 / 3$ independent on the curvature. If one neglects the source in the continuity equation (that is again possible for large $a$ or large $\Omega_{m}$ ), which consists of two terms, proportional to $H^{3}$ and $H \Lambda$ respectively, we come to the usual Friedmann-Lemaitre cosmology with the cosmological constant $\| \Lambda^{\prime}$.

The density parameter for $\Lambda$ term is

$$
\Omega_{\Lambda}=\frac{\Lambda}{3 H_{0}^{2}}\left(1-\frac{3 k}{\pi^{2}}\right)
$$

and the scale factor today is arbitrary quantity in this model.

I| Note that the cosmological constant appears naturally within McCrea-Milne approach [12]. 
Mass transfer rate (10) is simply

$$
\mathcal{M}=\frac{1}{\Omega_{m}}
$$

so it precisely cancels expansion with $\Omega_{m} \rightarrow 1$.

Model 3 (varying gravitational constant). We introduce the fundamental constant $\mu_{G X}=\frac{\Lambda}{8 \pi G}=$ const, the density of the vacuum. In this case the gravitational constant changes, $G=\frac{\pi}{8 \mu_{G X}}\left(\frac{c}{a}\right)^{2}$, while the speed of light does not. The cosmological term also acquires dependence on the scale factor through $G$ and is given by (21). Cosmological equations are

$$
\begin{aligned}
& H^{2}+\frac{k c^{2}}{a^{2}}=\frac{\pi^{2}}{3}\left(\frac{c}{a}\right)^{2}\left(1+\frac{\mu}{\mu_{G X}}\right), \\
& \dot{\mu}+3 H \mu=2 H \mu\left(1+\frac{\mu_{G X}}{\mu}\right),
\end{aligned}
$$

These are very different from the usual Friedmann equations. Even if could neglect the source terms in the continuity equation (that obviously cannot be done), the first cosmological equation does not correspond to the first Friedmann one. However, phase portrait of solutions for this model on the plane $\{\mu, a\}$ looks similar to the one of model 2 [8].

Again there is solution for density

$$
\mu=\mu_{0} \frac{a_{0}}{a}+2 \mu_{G X}\left(1-\frac{a_{0}}{a}\right) .
$$

Notice similarity with (15). This is due to the fact that $\Lambda$ is a constant in the model 2 , while $\mu_{G X}$ is constant in the model 3 , so the dependence of matter density on the scale factor is the same, although the dependence of dark energy on the scale factor is different.

Solution for the scale factor looks instead exactly the same as the solution for model 1, (12) with

$$
A_{3}=A_{1}, \quad B_{3}=\frac{\pi^{2}}{3 \mu_{G X}} c^{2} a_{0}\left(\mu_{0}-2 \mu_{G X}\right) .
$$

The separatrix is the same as in model 1 , given by $B_{3}=0$.

Matter density parameter for this model is

$$
\Omega_{m}=\frac{\pi^{2}}{3} \frac{c^{2}}{H_{0}^{2} a_{0}^{2}} \frac{\mu}{\mu_{G X}},
$$

that is the same as (17). The scale factor today is the same as in model 1 and is given by (14), as this expression follows from the definition of density parameter for curvature, which is the same as in model 1. Another, equivalent expression follows from definitions of vacuum mass density and critical density and it gives

$$
a_{0}^{2}=\frac{\pi c^{2}}{8 G_{0} \mu_{G X}} \text {. }
$$


Mass transfer rate (10) for this model is

$$
\mathcal{M}=\frac{2}{3 \Omega_{m}}
$$

that is analogous to the model 2 , but with $\Omega_{m} \rightarrow 1$ it corresponds to a constant fraction of expansion rate.

Model 4 (varying speed of light, constant vacuum energy density). The constant vacuum energy density is introduced as $\rho_{G X}=\frac{\Lambda c^{2}}{8 \pi G}=$ const, requiring variation of the speed of light $c=\left(\frac{8 G \rho_{G X}}{\pi}\right)^{1 / 4} a^{1 / 2}$. Here $G=$ const and $\Lambda=2 \sqrt{2} \pi^{3 / 2} \sqrt{G \rho_{G X}} a^{-1}=$ $\frac{3 \beta}{a\left(1-\frac{3 k}{\pi^{2}}\right)}$, where the constant $\beta$ appears in the cosmological equations

$$
\begin{aligned}
& H^{2}=\frac{8 \pi G}{3} \mu+\frac{\beta}{a}, \\
& \dot{\mu}+3 H \mu=\frac{3 H}{8 \pi G}\left(H^{2}+\frac{\beta}{a} \frac{\pi^{2}+3 k}{\pi^{2}-3 k}\right),
\end{aligned}
$$

with $\beta=\frac{2 \sqrt{2} \pi^{3 / 2}}{3}\left(G \rho_{G X}\right)^{1 / 2}\left(1-\frac{3 k}{\pi^{2}}\right)$.

The source term in the continuity equation can be neglected for large $a$, but the first Friedmann equation contains the positive $a^{-1}$ term which is again very different from the standard cosmology.

There is complete solution for this model with

$$
\mu=\mu_{0}\left(\frac{a_{0}}{a}\right)^{2}+\frac{1}{a} \sqrt{\frac{\pi \rho_{G X}}{2 G}}\left(1-\frac{a_{0}}{a}\right),
$$

and

$$
a=a_{0} \pm t \sqrt{A_{4}+a_{0} B_{4}}+\frac{B_{4}}{4} t^{2}
$$

where

$$
A_{4}=\frac{8 \pi G}{3} a_{0}\left(\mu_{0} a_{0}-\sqrt{\frac{\pi \rho_{G X}}{2 G}}\right), \quad B_{4}=2 \sqrt{\frac{2 G \rho_{G X}}{\pi}}\left(\pi^{2}-k\right) .
$$

This solution reduces to (13) for $B_{4}=0$ or, in other words, when $\Omega_{m} \rightarrow 1$. The separatrix is given by $\Omega_{m}=2 / 3$, like in model 2 .

Here new density parameter for dark energy is defined as

$$
\Omega_{\beta}=\frac{\beta}{H_{0}^{2} a_{0}},
$$

and the scale factor value today follows from this definition

$$
a_{0}=\frac{c_{0}}{H_{0}} \frac{\pi}{\sqrt{3\left(1-\Omega_{m}\right)}} .
$$

Notice that it is different from the scale factor in models 1 and 3 by the factor $\sqrt{1-3 k / \pi^{2}}$

Mass transfer rate (10) for this model is given by

$$
\mathcal{M}=\frac{1}{3 \Omega_{m}}+\left(1+\frac{3 k}{\pi^{2}}\right)\left(\frac{1}{\Omega_{m}}-1\right)
$$


from which we find that with $\Omega_{m} \rightarrow 0$ the vaccum again dominates expansion, while in the opposite case this is just a constant fraction of expansion rate. Therefore, it cannot be reduced to the usual Friedmannian model.

Finally, in the case 5 of [4] cosmological equations are given by the system (4)-(6). Definitions (71) and (8) can be used in this general case as well.

\subsection{Models with dark energy, dust and radiation}

So far we considered cosmological models based in GX formula (11), containing only dust and dark energy. In this subsection we turn to a more general class of models, including into consideration radiation.

It is known (e.g. 9]) that there is ambiguity in definition of multicomponent models with dark energy and/or variation of constants. There exist strong bounds on coupling between dark energy and radiation [13, [14, so we avoid this ambiguity assuming decoupled radiation, namely

$$
\rho_{r}=\rho_{r 0}\left(\frac{a}{a_{0}}\right)^{-4}, \quad p_{r}=\frac{\rho_{r}}{3}
$$

where the subscript " $r$ " refers to radiation. In standard cosmology any relativistic particle in thermodynamic equilibrium can be described by these equations. However, varying speed of light implies variation of dispersion relation $\epsilon^{2}=\Pi c^{2}+m_{0} c^{4}$, where $\epsilon$ and $\Pi$ are particle's energy and momentum and $m_{0}$ is its rest mass, hence influences

equation of state for ultrarelativistic fluid. In this paper we constrain ourselves with the case of thermal photons only.

Radiation density parameter in what follows is defined by

$$
\Omega_{r}=\frac{8 \pi G_{0}}{3 H_{0}^{2} c_{0}^{2}} \rho_{r 0}
$$

Then total energy conservation (6) implies

$$
\begin{aligned}
\frac{d \mu}{d a}+3 \frac{\mu}{a}=\frac{\Lambda}{8 \pi G}( & \left.2 \frac{d c}{c d a}-\frac{d \Lambda}{\Lambda d a}\right)+ \\
+ & {\left[\mu+\frac{\rho_{r 0}}{c^{2}}\left(\frac{a}{a_{0}}\right)^{-4}\right]\left(4 \frac{d c}{c d a}-\frac{d G}{G d a}\right)-2 \mu \frac{d c}{c d a} . }
\end{aligned}
$$

It is clear from (20) that in general matter couples to radiation always except for the case $c=G=$ const.

In this section we provide solutions for the set of cosmological models described by (41),(15),(18) and (20). Generally speaking, these models do not reduce to the ones considered above because of special role of radiation, given by (18).

Model 1, with radiation. This is the unique case when the radiation does not couple to matter density, because neither speed of light, nor the gravitational constant vary. 
Cosmological equations for this model read

$$
\begin{aligned}
& H^{2}+\frac{k^{\prime} c^{2}}{a^{2}}=\frac{8 \pi G}{3}\left(\mu+\frac{\rho_{r 0}}{c^{2}}\left(\frac{a}{a_{0}}\right)^{-4}\right), \\
& \dot{\mu}+3 H \mu=\frac{\pi}{4 G}\left(\frac{c}{a}\right)^{2} H,
\end{aligned}
$$

Solution for the matter density is the same (111) as it is in the absence of radiation.

Additional peculiarity of this case is the presence of separatrix, playing the same role as for models without radiation. The separatrix is defined by

$$
\Omega_{m}=\frac{2}{3} \frac{1-\Omega_{r}}{1-\frac{k}{\pi^{2}}},
$$

that again gives $\Omega_{m} \approx 2 / 3$ for any curvature parameter.

Implicit solution for the scale factor as a function of cosmic time is

$$
t-t_{0}=\frac{1}{\tilde{A}_{2}}\left(\sqrt{F(a)}-\sqrt{F\left(a_{0}\right)}\right)-\frac{\tilde{A}_{1}}{2 \tilde{A}_{2}^{\frac{3}{2}}} \log \left(\frac{\tilde{A}_{1}+2 a \tilde{A}_{2}+2 \sqrt{\tilde{A}_{1} F(a)}}{\tilde{A}_{1}+2 a_{0} \tilde{A}_{2}+2 \sqrt{\tilde{A}_{1} F\left(a_{0}\right)}}\right),
$$

where

$$
F(x)=\tilde{A}_{0}+\tilde{A}_{1} x+\tilde{A}_{2} x^{2}
$$

and

$$
\begin{aligned}
& \tilde{A}_{0}=\frac{8 \pi G}{3 c^{2}} \rho_{r 0} a_{0}^{4}, \\
& \tilde{A}_{1}=\frac{8 \pi G}{3} a_{0}\left(\mu_{0} a_{0}^{2}-\frac{\pi c^{2}}{4 G}\right), \\
& \tilde{A}_{2}=\left(\pi^{2}-k\right) c^{2} .
\end{aligned}
$$

The solution for separatrix is explicit and looks rather simple

$$
a=\frac{c}{H_{0}} \sqrt{\frac{\pi^{2}-k}{1-\Omega_{r}}} \sqrt{1+2 H_{0} t+\left(1-\Omega_{r}\right) H_{0}^{2} t^{2}} .
$$

This solution approaches (13) at late times. Solution for density is represented at fig. (1I) for selected values of $\Omega_{m}$. Like in models without radiation solutions with $\Omega_{m}>2 / 3$ reach Friedmannian singularity in the past, while solutions with $\Omega_{m}<2 / 3$ start with vanishing density.

Both density parameter and critical density are defined by (17). Radiation density parameter is as usual (19). Curvature density parameters remains the same as in model without radiation, and the scale factor becomes a little bigger due to the change $\sqrt{1-\Omega_{m}} \rightarrow \sqrt{1-\Omega_{m}-\Omega_{r}}$ in (14).

Model 2, with radiation. Since the speed of light varies in this model, matter becomes coupled to radiation and solution gets complicated. 


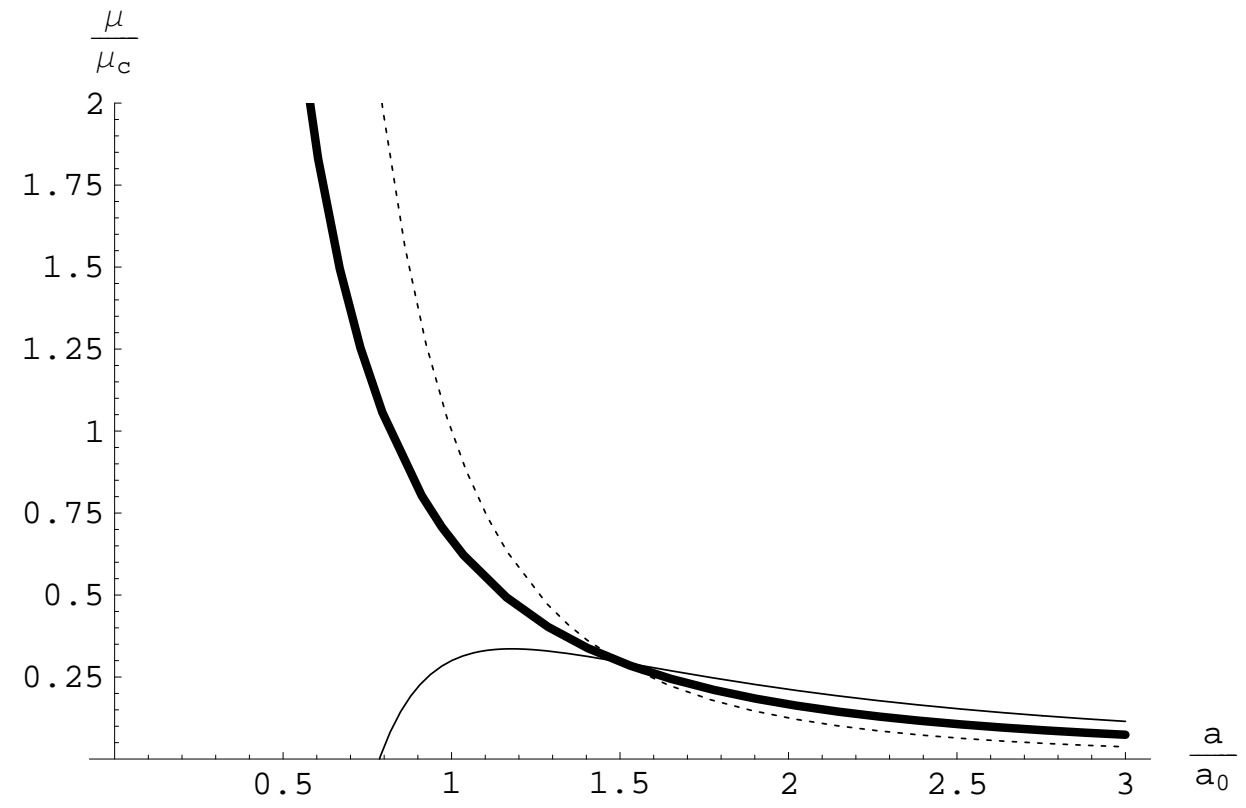

Figure 1. Density of matter in model 1 in terms of critical density, depending on the scale factor in terms of present scale factor with $\Omega_{m}$ equal to 0.3 (firm curve), 0.667 (thick curve) and 0.999 (dotted curve).

Cosmological equations for this model are

$$
\begin{aligned}
& H^{2}-\frac{\Lambda^{\prime}}{3}=\frac{8 \pi G}{3}\left(\mu+\frac{\pi^{2}}{\Lambda} \rho_{r 0} \frac{a_{0}^{4}}{a^{6}}\right), \\
& \dot{\mu}+H\left(\mu-\frac{\Lambda}{4 \pi G}\right)=\frac{4 \pi^{2} \rho_{r 0} a_{0}^{4}}{\Lambda} \frac{H}{a^{6}} .
\end{aligned}
$$

Hence the solution for the density is

$$
\mu(a)=\frac{1}{a}\left[\frac{\Lambda}{4 \pi G}\left(a-a_{0}\right)+\mu_{0} a_{0}+\frac{4 \pi^{2}}{5 \Lambda a_{0}} \rho_{r 0}\left(1-\left(\frac{a}{a_{0}}\right)^{-5}\right)\right] .
$$

This model does not possess separatrix in the sense discussed above, because the early expansion is dominated by the negative definite term (since the vacuum energy is positive definite). The solution for matter density in this model is represented at fig. (2).

Although there is no separatrix with respect to initial singularity, the value $\Omega_{m}=2 / 3$ again plays important role, dividing solutions in two classes, depending on the sign of time derivative of the density: for $\Omega_{m}>2 / 3$ the density decreases with time in matter dominated epoch, like in Friedmannian models, while for $\Omega_{m}<2 / 3$ the density increases with time. This behavior is seen from equation for the density, since the condition $\Lambda=4 \pi G \mu$ leads to $\Omega_{m}=2 / 3$.

In contrast to simple GX model without radiation, the scale factor is no more arbitrary in this model and is given by (14) with the change $\sqrt{1-\Omega_{m}} \rightarrow \sqrt{1-\Omega_{m}-\Omega_{r}}$. 


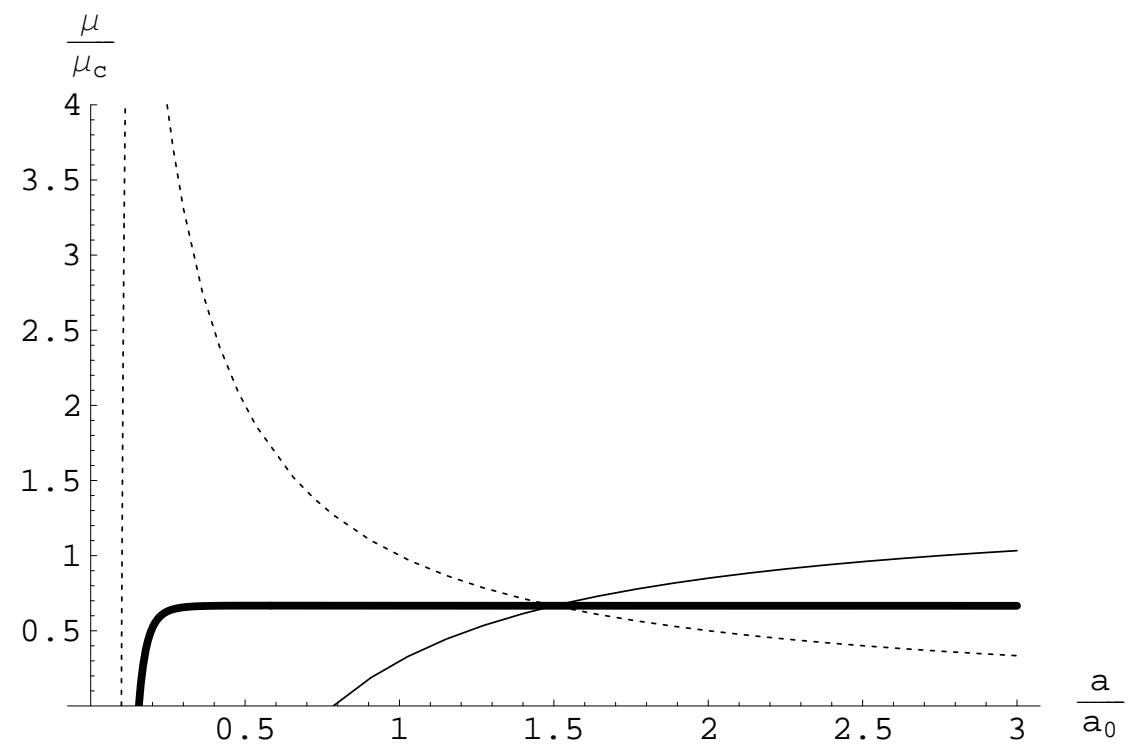

Figure 2. Density of matter in model 2. $\Omega_{m}$ equals to 0.3 (firm curve), 0.667 (thick curve) and 0.999 (dotted curve).

Radiation density parameter is

$$
\Omega_{r}=\frac{\pi^{2}}{\Lambda} \frac{\rho_{r 0}}{\mu_{c} a_{0}^{2}}
$$

and it coincides with (19).

Model 3, with radiation. Cosmological equations now reduce to

$$
\begin{aligned}
& H^{2}+\frac{k c^{2}}{a^{2}}=\frac{2 \pi^{2}}{3}\left(\frac{c}{a}\right)^{2}\left[1+\frac{1}{\mu_{G X}}\left(\mu+\frac{\rho_{r 0}}{c^{2}}\left(\frac{a}{a_{0}}\right)^{-4}\right)\right], \\
& \dot{\mu}+H\left(\mu-2 \mu_{G X}\right)=2 H \frac{\rho_{r 0}}{c^{2}}\left(\frac{a}{a_{0}}\right)^{-4},
\end{aligned}
$$

Solution for density in this model is

$$
\mu(a)=2 \mu_{G X}+\frac{a_{0}}{a}\left(\mu_{0}-2 \mu_{G X}\right)+\frac{2}{3} \frac{\rho_{r 0}}{c^{2}} \frac{a_{0}}{a}\left(1-\left(\frac{a_{0}}{a}\right)^{3}\right),
$$

and its dependence on the scale factor is similar to the one shown at fig. (2). This is because again with negligible contribution of radiation the time derivative of the density is determined by the condition $\mu=2 \mu_{G X}$ which leads to the same density parameter $\Omega_{m}=2 / 3$.

The scale factor is the same as in the model 2 above. Radiation density parameter is

$$
\Omega_{r}=\frac{\pi^{2}}{3} \frac{\rho_{r 0}}{\mu_{G X} a_{0}^{2} H_{0}^{2}},
$$

and it coincides with (19) due to definition of $\mu_{G X}$. 
Model 4, with radiation. For this case cosmological equations read

$$
\begin{aligned}
& H^{2}=\frac{8 \pi G}{3}\left(\mu+\frac{1}{a} \sqrt{\frac{\pi}{8 G \rho_{G X}}} \rho_{r 0}\left(\frac{a}{a_{0}}\right)^{-4}\right)+\frac{\beta}{a}, \\
& \dot{\mu}+2 H \mu=\frac{H}{a} \sqrt{\frac{\pi \rho_{G X}}{2 G}}\left(1+\frac{\rho_{r 0}}{\rho_{G X}}\left(\frac{a}{a_{0}}\right)^{-4}\right),
\end{aligned}
$$

The solution for model 4 is

$$
\mu(a)=\mu_{0}\left(\frac{a_{0}}{a}\right)^{2}+\sqrt{\frac{\pi \rho_{G X}}{2 G}} \frac{1}{a}\left(1-\frac{a_{0}}{a}\right)+\frac{\rho_{r 0} a_{0}}{3} \sqrt{\frac{\pi}{2 G \rho_{G X}}} \frac{1}{a^{2}}\left(1-\left(\frac{a_{0}}{a}\right)^{3}\right),
$$

and it is represented at fig. (3).

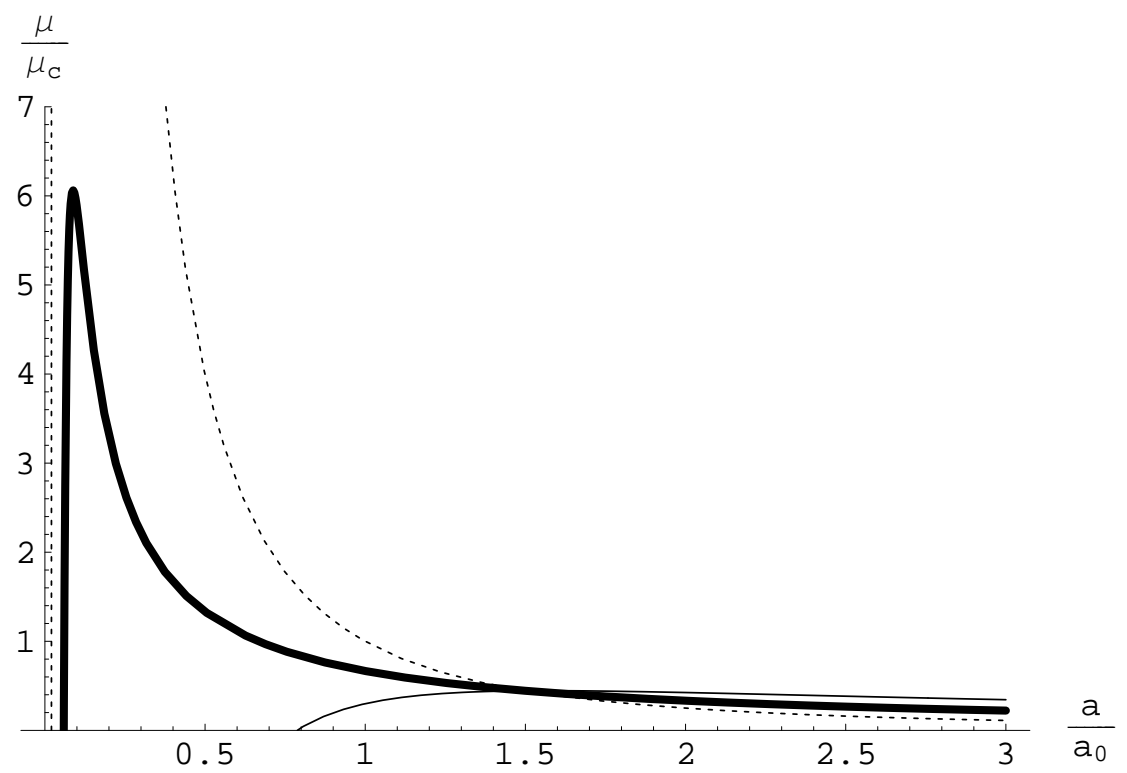

Figure 3. Density of matter in model $4 . \Omega_{m}$ equals to 0.3 (firm curve), 0.667 (thick curve) and 0.999 (dotted curve).

There is no special value of density parameter in model 4. Solutions start with zero density and positive scale factor, then the density increases rapidly in radiation dominated stage, but entering into matter dominated stage it starts to decrease, so for each solution there is a maximum in the density (see fig. (3)).

Here the density parameter of radiation is defined by

$$
\Omega_{r}=\frac{16 \pi^{3 / 2}}{3 H_{0}^{2} a_{0}} \sqrt{\frac{G}{\rho_{G X}}} \rho_{r 0},
$$

and it is again equivalent to (19). 


\section{Discussion and conclusions}

In this paper we explored various cosmological models with Gurzadyan-Xue dark energy formula (11), assuming also variation of physical constants such as the speed of light and the gravitational constant. It is shown that unlike standard Friedmann models, various interesting possibilities appear. In particular in the model 1 the continuity equation is reduced to the energy transfer from dark energy component into the usual matter. For

some cases (models 1 and 4), the source term in the continuity equation can be neglected for sufficiently large scale factors (or sufficiently large present density of matter), and the discussion of the dynamics in this asymptotic cases can be given in terms of the standard cosmology.

Solutions for the density in terms of the scale factor for all models with pressureless matter and dark energy contain separatrix which divides solutions in two classes. The first class is analogous to the standard Friedmann solutions with singularity in the beginning of expansion. The second class, in contrast, does not contain singularity; solutions start with vanishing density and non-zero scale factor.

We also considered generalization of GX models, including radiation, assuming that dynamics of the latter is not influenced by the variation of constants and by dark energy. Interestingly, for the model 1 we obtained the behavior qualitatively similar to models without radiation. For the rest of models separatrix in the sense discussed above is absent and all solutions belong to the second class, namely non-Friedmannian solutions. The density in these solutions may be larger in the past, but if so, it has a maximum and all solutions start with vanishing density in radiation-dominated epoch. However for models 2 and 3 with radiation the same value of density parameter $\Omega_{m}=2 / 3$ that parametrizes separatrix in simple GX models, corresponds to constant mass density of pressureless matter in matter dominated epoch. For larger density parameter the mass density decreases with expansion and for smaller density parameter it increases.

In fact, not all GX models with non-Friedmannian behavior $\left(\Omega_{m}<2 / 3\right)$ pass age constraint and have deceleration parameter which fits the value followed from observations. At the same time model IV with $\Omega_{m} \geq 2 / 3$ pass both constraints and represent a good candidate for dark energy model [7] which in the limit of vanishing energy transfer from dark energy to the usual matter has $a^{-1}$ term in Friedmann equation. In the same way models I and III fit these constraints for density parameter slightly smaller than the separatrix value. This circumstance once more points out on a special role of the separatrix in GX models.

Notice another interesting feature of GX models with radiation. It is well known that Friedmannian models with zero curvature have arbitrary scale factor. The same holds also for simple GX models (model 2). However, this degeneracy breaks down in GX models with radiation, where the scale factor can always be computed in terms of present density parameters, and present values of (varying) physical constants.

We are thankful to the referees for helpful comments. 


\section{References}

[1] V. G. Gurzadyan, S.-S. Xue in: "From Integrable Models to Gauge Theories; volume in honor of Sergei Matinyan", ed. V. G. Gurzadyan, A. G. Sedrakian, p.177, World Scientific, 2002;Mod. Phys. Lett. A18 (2003) 561 astro-ph/0105245

[2] V. G. Gurzadyan, S.-S. Xue, astro-ph/0510459

[3] Ya. B. Zeldovich JETP Lett. 6 (1967) 883; Sov. Phys. - Uspekhi 95 (1968) 209.

[4] G.V. Vereshchagin, Mod. Phys. Lett. A21 (2006) 729.

[5] M. Duff, L.B. Okun and G. Veneziano, JHEP, 03 (2002) 023.

[6] J. Magueijo, Rept.Prog.Phys., 66 (2003) 2025; J.D. Barrow, D. Kimberly and J. Magueijo, Class.Quant.Grav., 21 (2004) 4289.

[7] G.V. Vereshchagin, G. Yegorian, astro-ph/0604566, submitted for publication to Phys. Lett. B.

[8] G.V. Vereshchagin, G. Yegorian, Phys. Lett. B636 (2006) 150.

[9] T. Harko and M. K. Mak, Class. Quantum Grav. 16 (1999) 2741.

[10] A. Albrecht, J. Magueijo, Phys.Rev., D59 (1999) 043516.

[11] I.L. Shapiro, J. Solà and H. Štefančić, JCAP, 01 (2005) 012.

[12] V.G. Gurzadyan, The Observatory 105 (1985) 42.

[13] R. Opher, A. Pelinson, Phys. Rev. D70 (2004) 063529.

[14] R. Opher, A. Pelinson, MNRAS 362 (2005) 167. 